\title{
ARPP protein is selectively expressed in renal oncocytoma, but rarely in renal cell carcinomas
}

\author{
Kohei Shomori ${ }^{1}$, Yoji Nagashima ${ }^{2}$, Naoto Kuroda ${ }^{3}$, Ami Honjo ${ }^{1}$, Yoshiyuki Tsukamoto ${ }^{4}$, \\ Naruo Tokuyasu $^{1}$, Noritaka Maeta ${ }^{1}$, Keiko Matsuura ${ }^{5}$, Naoki Hijiya ${ }^{5}$, Shinji Yano ${ }^{4}$, \\ Shigeo Yokoyama ${ }^{4}$, Hisao Ito $^{1}$ and Masatsugu Moriyama ${ }^{4}$ \\ ${ }^{1}$ Faculty of Medicine, Division of Organ Pathology, Department of Microbiology and Pathology, Tottori \\ University, Yonago, Japan; ${ }^{2}$ Department of Molecular Pathology, Yokohama City University Graduate School \\ of Medicine, Yokohama, Japan; ${ }^{3}$ Department of Pathology, Kochi Red Cross Hospital, Kochi, Japan; ${ }^{4}$ Faculty \\ of Medicine, Department of Human Pathology, Oita University, Oita, Japan and ${ }^{5}$ Faculty of Medicine, \\ Department of Molecular Pathology, Oita University, Oita, Japan
}

\begin{abstract}
We have recently isolated a gene, Ankyrin-repeated protein with a proline-rich region (ARPP), that is highly expressed in the skeletal and cardiac muscle. Our previous immunohistochemical analysis revealed that ARPP expression was augmented in rhabdomyosarcoma but scarcely detectable in leiomyosarcoma, showing that ARPP is a useful marker for rhabdomyosarcoma. In the present study, we generated the anti-ARPP monoclonal antibody, YAS11, immunoreactive with the $\mathrm{N}$-terminal region (amino-acids residues 1-145) of the ARPP protein. Further, we immunohistochemically analyzed 100 renal tumors including 14 oncocytomas, and 86 renal cell carcinomas (RCCs). We found that ARPP was highly expressed in 12 of the $14(85.7 \%)$ oncocytomas, but was detectable in only four of the $86(4.7 \%)$ RCCs. Interestingly, ARPP was not detected in any of 11 chromophobe RCCs, suggesting that ARPP may be useful for differential diagnosis between oncocytoma and chromophobe RCC. Furthermore, we found that ARPP was selectively expressed in part of the distal renal tubule in normal kidney. Immunoelectron microscopy with anti-ARPP antibody revealed that ARPP was localized in mitochondria and nuclei in both the normal distal renal tubule and oncocytoma, suggesting that oncocytoma may be derived from the distal nephron, and probably from part of the distal renal tubule.
\end{abstract}

Modern Pathology (2007) 20, 199-207. doi:10.1038/modpathol.3800730; published online 5 January 2007

Keywords: ARPP; chromophobe RCC; renal oncocytoma

Ankyrin-repeated protein with a proline-rich region $(A R P P)$ was originally identified in our laboratory as a protein that is highly expressed in esophageal carcinoma cells. ${ }^{1}$ Another group also identified a murine counterpart of ARPP, Ankrd2, as a protein that is inducible in the stretched skeletal muscle. ${ }^{2}$ ARPP is composed of 333 amino acids and is characterized by the presence of four ankyrin-like repeat motifs in its middle portion and PEST-like sequences in the amino-terminal regions. PEST sequences are rich in proline (P), aspartic and glutamic acids (E), serine (S) and threonine (T),

Correspondence: Dr K Shomori, MD, PhD, Faculty of Medicine, Division of Organ Pathology, Department of Microbiology and Patholgy, Tottori University, Nishi-cho 86, Yonago-shi, Tottori, 683-8503, Japan.

E-mail: shomori@grape.med.tottori-u.ac.jp

Received 5 August 2006; revised 17 October 2006; accepted 23 October 2006; published online 5 January 2007 and are, in many cases, implicated in the regulation of protein turnover. However, immunohistochemical analysis of a large number of esophageal carcinomas revealed that the expression level of ARPP was relatively lower in clinical samples than in esophageal carcinoma cell lines. ${ }^{1}$ Unexpectedly, ARPP was found to be expressed at a high level in almost all rhabdomyosarcomas. ${ }^{3,4}$ On the other hand, in normal tissues, we found that ARPP was preferentially expressed in skeletal and cardiac muscle. ${ }^{1,3}$ Moreover, ARPP was abandunt in mouse denervated gastrocnemius muscles, ${ }^{5}$ whereas the biological function of ARPP is largely unknown. In the present study, we found that ARPP was expressed in part of the distal tubule of the kidney, and also expressed specifically in renal oncocytoma, a benign tumor of the kidney. As ARPP was barely detectable in the granular form of conventional renal cell carcinoma or in the eosinophilic variant of chromophobe cell carcinoma, both of which are 
sometimes difficult to distinguish histopathologically from oncocytoma, ARPP may have potential use as a marker for distinguishing oncocytoma from other renal tumors.

\section{Materials and methods}

\section{Generation of Anti-ARPP Monoclonal Antibody}

Amino acids 5-333 of the full-length ARPP protein (333 amino acids) fused to glutathione-S-transferase (GST) was synthesized in Escherichia coli and purified as described previously. ${ }^{1}$ GST-ARPP protein $(20 \mu \mathrm{g})$ emulsified in complete Freund's adjuvant (Sigma, Tokyo, Japan) was injected into female BDF1 mice (Nippon Crea Inc., Tokyo, Japan) intraperitoneally (i.p.). The mice were given a booster injection of GST-ARPP protein emulsified in incomplete Freund's adjuvant (Sigma) i.p. at 2-week intervals. Three days after the final i.p. boost of GST-ARPP protein in an aqueous solution, spleen cells collected from each mouse were fused with a mouse myeloma cell line, SP2/0-Ag14, using polyethylene glycol 1300-1600 (Sigma), as described by Galfre and Milstein. ${ }^{6}$ The hybridomas producing anti-ARPP antibodies were screened with an enzyme-linked immunosorbent assay (ELISA) in microtiter plates pre-coated with GST-ARPP. We selected the most immunoreactive clone, which was designated as YAS11.

\section{Plasmids}

Expression plasmids encoding the full-length ARPP protein and its deletion mutants (see Figure 1b) were generated as follows. First, ARPP cDNAs encoding full-length ARPP and a series of deletion mutants were amplified by polymerase chain reaction (PCR) with the following primer pairs using pcDNA3ARPP plasmid ${ }^{1}$ as a template: Arp-f1; $5^{\prime}$-TCTCGA GATGGAGGACTCCGAGGCGGTG- $3^{\prime}$ and Arp-r1; 5'-TGCGGCCGCACTGGGCTGGCACAGGCTG-3' ${ }^{\prime}$ for the full-length ARPP (1-333), Arp-f1 and Arp-r2; $5^{\prime}$-TGCGGCCGCTCTTTCCTGCCAGGTTCTT-3' ${ }^{\prime}$ for ARPP (1-287), Arp-f1 and Arp-r4; 5'-TGCGGCCGC
ACGTGTCGGCTGACCCCCC-3' for ARPP (1-145), Arp-f2; 5'-TCTCGAGAAGAAACGCAAGCAGAAG AAGCG-3' and Arp-r1 for ARPP (93-333). Next, each PCR product was subcloned into the pGEM-T Easy vector (Promega, Madison, WI, USA), followed by sequencing. Finally, the cloned cDNAs were each digested with XhoI/Not1 and inserted into the pMe-flag vector, which expresses flag-tagged proteins. $^{7}$

\section{Enzyme-Linked Immunosorbent Assay}

ELISA was performed according to the methods described previously. ${ }^{8}$ Briefly, microtiter plates were coated overnight with $50 \mu \mathrm{l}$ of each GST-ARPP solution containing $1 \mu \mathrm{g} / \mathrm{ml}$ GST-ARPP and $0.1 \%$ sodium azide in PBS at $4^{\circ} \mathrm{C}$, followed by blocking with $2 \%$ bovine serum albumin (Sigma) in PBS at $4^{\circ} \mathrm{C}$. Then, the titer of each antibody was determined as described previously. ${ }^{8}$

\section{Tissue Samples}

The paraffin-embedded tissues used in this study were obtained at the time of surgery for renal tumors. Tissue samples from 100 renal tumors were selected from the files of the Division of Organ Pathology, Tottori University, the Department of Molecular Pathology, Yokohama City University, and the Department of Pathology, Kochi University. The specimens comprised 29 clear cell-type renal cell carcinomas (RCCs), 39 cases of the eosinophilic variant of clear cell-type RCCs, 11 chromophobe RCCs, seven papillary RCCs (four Type I and three Type II), and 14 oncocytomas, which had been diagnosed histologically according to the WHO classification. ${ }^{9}$ Eosinophilic variant of clear cell-type RCCs were identified by nuclear atypia, immunohistologically positive with vimentin and epithelial membranous antigen (EMA), and no staining reaction with Hale's collidal iron stain. Rarer types of the RCC (sarcomatoid type etc) were not included in this research. Samples of normal control kidney tissue were obtained at autopsy from two patients who had
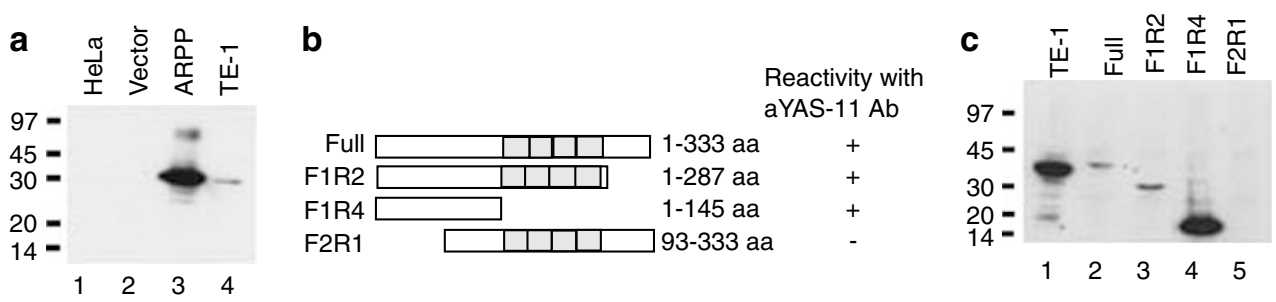

Figure 1 Characterization of the anti-human ARPP monoclonal antibody, YAS11. (a) Western blot analysis of ARPP-transfected HeLa cells. HeLa cells transfected with the pcDNA3-Arpp plasmid (lane 3), pcDNA3 vector alone (lane 2), or without plasmid (lane 1) were analyzed by Western blotting for expression of ARPP. TE-1 cells that endogenously express ARPP were also analyzed by Western blotting (lane 4). (b) Schematic representation of a series of deletion constructs encoding flag-ARPP(1-333), flag-ARPP(1-287), flag-ARPP(1-145), and flag-ARPP(93-333). (c) HeLa cells transfected with flag-ARPP(1-333), flag-ARPP(1-287), flag-ARPP(1-145), and flag-ARPP(93-333) were analyzed for ARPP expression by Western blotting with YAS-11 Ab. 
died of non-kidney-related disease. Normal kidney tissue taken from areas adjacent to kidney tumors was also used as a control. Use of these tissue samples for the present study was approved by the Institutional Review Board of Tottori University (permission no. 2001-149).

\section{Transfection}

HeLa cells were grown in Dulbecco's-modified Eagle medium (DMEM), containing 10\% heat-inactivated fetal bovine serum (FBS) (JRH Biosciences, Lenexa, $\mathrm{KS}$, USA), $100 \mathrm{U} / \mathrm{ml}$ penicillin, $100 \mu \mathrm{g} / \mathrm{ml}$ streptomycin and $2 \mathrm{mM}$ glutamine. The cells were transfected with expression plasmids using Lipofectamine $^{\mathrm{TM}} 2000$ (Invitrogen, Carlsbad, CA, USA) in Opti-MEM I medium (Invitrogen) as recommended by the manufacturer. At $6 \mathrm{~h}$ after transfection, the cells were further cultured in $1 \mathrm{ml}$ of DMEM without serum. Transient protein expression was monitored by Western blot analysis (see below).

\section{Immunohistochemistry}

Tissue sections were cut from paraffin-embedded tissue at a thickness of $4 \mu \mathrm{m}$ and deparaffinized in xylene, followed by quenching of endogenous peroxidase using $3 \% \mathrm{H}_{2} \mathrm{O}_{2}$ in methanol for $30 \mathrm{~min}$ at room temperature. After a thorough wash in water, the sections were microwaved for antigen retrieval in $0.01 \mathrm{M}$ citrate buffer at $92^{\circ} \mathrm{C}$ for $20 \mathrm{~min}$. The sections were allowed to cool before being washed in $1 \times$ PBS, and they were then incubated with $2 \%$ (FBS) in PBS for blocking. Subsequently, the sections were incubated for $1 \mathrm{~h}$ with primary monoclonal antibodies against ARPP (YAS11), EMA (Nichirei, Tokyo, Japan), CD10 (Nichirei), and MUC1 (Novocastra, Newcastle-upon-Tyne, UK). Negative controls were prepared by omitting the primary antibody. The sections were washed with $1 \times$ PBS three times, and then incubated with the second antibody, horseradish peroxidase-labeled rabbit anti-mouse IgG antiserum (Nichirei, Japan), for $30 \mathrm{~min}$ at RT. After reaction with a $\mathrm{H}_{2} \mathrm{O}_{2} / 3,3^{\prime}$ diaminobenzidine substrate solution, the sections were counterstained with hematoxylin and mounted. For immunohistochemical evaluation, each case was considered positive when $10 \%$ or more of the tumor cells were positively stained, and as negative when $<10 \%$ of the tumor cells positively stained.

\section{Double-Labeled Immunofluorescence}

Paraffin-embedded tissue sections were deparaffinized, immersed in $10 \mathrm{mM}$ sodium citrate buffer (pH 6.0) (Wako, Osaka, Japan), and microwaved at $92^{\circ} \mathrm{C}$ for $20 \mathrm{~min}$. Subsequently, they were blocked with $10 \%(\mathrm{v} / \mathrm{v})$ normal goat serum (Nichirei Co.) for
$30 \mathrm{~min}$ at RT. The sections were then incubated for $18 \mathrm{~h}$ at $4{ }^{\circ} \mathrm{C}$ with a mixture of ARPP rabbit polyclonal $\mathrm{Ab}$ diluted 1:1000 ${ }^{1}$ and MUC-1 Ab diluted 1:50, or pre-diluted CD10. After being washed with PBS, they were incubated for $2 \mathrm{~h}$ at RT with a mixture of Alexa Fluor 488-conjugated goat anti-rabbit antibody diluted 1:200 and Alexa Fluor 546-conjugated goat anti-mouse antibody (Molecular Probes Inc., Eugene, OR, USA) diluted 1:200 in PBS. The sections were then washed with PBS and mounted with gel/mount (Biomeda Corp., Foster City, CA, USA). The mounted sections were observed with a fluorescence microscope (Eclipse E800; Nikon, Tokyo, Japan), and the images were processed with the MRC-1024 confocal system (Bio-Rad Laboratories Inc., Hercules, CA, USA).

\section{Western Blot Analysis}

Cells were lysed in SDS-modified RIPA buffer $(0.1 \%$ w/v SDS, $40 \mathrm{mM}$ 2-[4-(2-hydroxyethyl)-1-piperazinyl]ethanesulfonic acid (HEPES)- $\mathrm{NaOH}$ (pH 7.4), $1 \% \mathrm{v} / \mathrm{v}$ Nonidet P-40 (NP40), $0.5 \% \mathrm{w} / \mathrm{v}$ sodium deoxycholate, $150 \mathrm{mM} \mathrm{NaCl}, 1 \mathrm{mM}$ phenylmethylsulfonyl fluoride (PMSF), $10 \mathrm{mM}$ sodium pyrophosphate, $10 \mathrm{mM}$ sodium fluoride, $4 \mathrm{mM}$ ethylenediamine-tetraacetic acid (EDTA), and $2 \mathrm{mM}$ sodium vanadate) on ice for $10 \mathrm{~min}$ and centrifuged at 15000 r.p.m. at $4^{\circ} \mathrm{C}$ for $20 \mathrm{~min}$. The resulting cell lysates $(50 \mu \mathrm{g})$ were boiled with Laemmli sample buffer and then subjected to SDS $(10 \% \mathrm{w} / \mathrm{v})-$ polyacrylamide gel electrophoresis. The samples were transferred to a polyvinylidene difluoride (PVDF) membrane (Millipore, Bedford, MA, USA), which was blocked for $1 \mathrm{~h}$ in $10 \% \mathrm{w} / \mathrm{v}$ skim milk in Tris-buffered saline (TBS) at $4^{\circ} \mathrm{C}$ and then incubated for $1 \mathrm{~h}$ with $1 \mu \mathrm{g} / \mathrm{ml}$ YAS11 Ab at RT. After the filter had been thoroughly washed with $1 \times$ TBS containing $0.1 \% \mathrm{v} / \mathrm{v}$ Tween 20 , it was incubated for $1 \mathrm{~h}$ at $4^{\circ} \mathrm{C}$ with goat anti-mouse IgG Fc-specific HRPconjugated antibody (Zymed Laboratories Inc., San Francisco, CA, USA). After a further wash with TBS containing $0.1 \% \mathrm{v} / \mathrm{v}$ Tween 20 , the signals were detected using the ECL Western blotting analysis system (Amersham Bioscience, Piscataway, NJ, USA) according to the manufacturer's instructions.

\section{Immunoelectron Microscopy}

Preparation of tissue samples

Tissue specimens of oncocytoma were prepared from paraffin-embedded blocks. A small piece of tumor tissue was cut out from the block and deparaffinized by immersion in xylene. After rehydration of the tissue samples in ethanol, they were washed in $2 \mathrm{M}$ phosphate buffer, $\mathrm{pH}$ 7.4. Subsequently, they were fixed in $2 \%$ glutaraldehyde in $0.2 \mathrm{M}$ phosphate buffer ( $\mathrm{pH} 7.4$ ) at $4^{\circ} \mathrm{C}$ for $4 \mathrm{~h}$, and post-fixed in $1 \% \mathrm{OsO}_{4}$ at $4^{\circ} \mathrm{C}$ for $2 \mathrm{~h}$. After dehydration in ethanol and propylene oxide, the samples 
were infiltrated with epoxy resin (Epok 812; Okenshoji Co., Tokyo, Japan) in propylene oxide, and finally embedded in Epok 812 with DMP-30 (TAAB Laboratories, Berkshire, UK).

The specimens of normal kidney tissue obtained from autopsy cases were cut into small pieces and then fixed directly in $2 \%$ glutaraldehyde in $0.2 \mathrm{M}$ phosphate buffer ( $\mathrm{pH} 7.4)$ at $4^{\circ} \mathrm{C}$ overnight. They were then processed in a similar way to the tissues prepared from oncocytomas.

Ultrathin sections were cut with an ultramicrotome (Leica Instruments, Nussloch, Germany) at a thickness of $90 \mathrm{~nm}$ and mounted on nickel grids that had been coated with carbon by vacuum evaporation.

\section{Immunostaining for ultrastructural localization of ARPP}

Yano et $a 1^{10}$ have recently developed a new method of antigen retrieval for immunoelectron-microscopy. The merit of this method is that it can be applied to ultrathin sections cut from routinely prepared epoxy resin-embedded blocks. ${ }^{10}$ The details of the method have already been reported..$^{10}$ Briefly, the ultrathin sections were microwaved in diluted Target Retrieval Solution (TRS) (DAKO, Carpinteria, CA, USA) at $\mathrm{pH} 10$ for $15 \mathrm{~min}$, followed by further incubation in TRS at room temperature for $30 \mathrm{~min}$. After incubation in blocking reagent (DAKO) for $10 \mathrm{~min}$, the sections were incubated for $60 \mathrm{~min}$ at $60^{\circ} \mathrm{C}$ with rabbit anti-ARPP polyclonal antibody, whose specificity for ARPP has been characterized in detail previously. ${ }^{3}$ Subsequently, they were washed in $1 \times$ TBS containing $0.1 \%$ BSA and $0.1 \%$ Tween 20 , and then incubated with goat anti-rabbit IgG antibody conjugated with gold particles $15 \mathrm{~nm}$ in diameter (Amersham Bioscience) for $30 \mathrm{~min}$ at $60^{\circ} \mathrm{C}$. After washing in TBS containing $0.1 \%$ BSA and $0.1 \%$ Tween 20, the sections were counterstained with uranyl acetate and citrate lead in a similar way to the routine procedure.

\section{Results}

\section{Specificity of the Anti-ARPP Monoclonal Antibody, YAS11, for ARPP}

To generate the anti-ARPP monoclonal antibody, we selected seven clones that produce antibodies immunoreactive with GST-ARPP as described in Materials and methods. To exclude clones that produced antibodies recognizing GST but not ARPP, we tested all the clones for immunoreactivity with GST by ELISA. As a result, we found that only one clone, designated YAS11, was immunoreactive with GST-ARPP and not with GST. To determine the specificity of the YAS11 Ab, HeLa cells transfected with pcDNA3-ARPP were analyzed by Western blotting with YAS11 Ab. As shown in Figure 1a, a single $43-\mathrm{kDa}$ band, corresponding to ARPP, was detected in cells transfected with pcDNA3-ARPP, whereas no bands were detectable in cells transfected with the vector alone (Figure 1a). Furthermore, a band of the same size was also detected in TE-1 cells that endogenously express ARPP. These findings indicated that YAS11 Ab can detect ARPP specifically. Next, to determine which part of the ARPP protein is immunoreactive with YAS11, we transfected a series a deletion constructs encoding truncated ARPP into HeLa cells (Figure 1b). The transfected cells were then analyzed by Western blot analysis with YAS11 Ab. As shown in Figure 1c, a single band was detectable in cells transfected with flag-ARPP(1-333), flag-ARPP(1-287) and flagARPP(1-145), but undetectable in cells transfected with flag-ARPP(93-333), suggesting that YAS11 may be immunoreactive with the N-terminal region (amino-acids residues 1-93).

\section{Selective Expression of ARPP in Part of the Distal Renal Tubule}

We then analyzed the expression of ARPP by immunohistochemistry with the YAS11 Ab. Consistent with our previous data obtained using rabbit polyclonal $\mathrm{Ab}, 3,5$ ARPP was found to be highly expressed in skeletal muscle (data not shown). In addition, we found unexpectedly that ARPP was expressed in part of the renal tubule (Figure 2a). Morphologically, the ARPP positivity in tubules, which are composed of cuboidal epithelial cells, was distributed in both the renal cortex and medulla (Figure 2a(B, C, E, and F)). In the renal cortex, the proximal tubule, composed of high columnar epithelial cells, was evidently negative for ARPP, but some parts of the renal tubule composed of low cuboidal epithelial cells were found to be positively immunostained (Figure 2a(B)). These morphological features suggest that the area of ARPP positivity may correspond to the distal renal tubule. Similar histochemical staining to that seen using YAS11 $\mathrm{Ab}$ was also observed in a study using a polyclonal antibody (data not shown). To further confirm the specific localization of the immunostaining, doubleimmunohistochemistry with anti-ARPP Ab and antiCD10 Ab, which is known to be immunoreactive with the proximal renal tubule, was performed. As shown in Figure 2b, the ARPP-positive part of the tubule did not coincide with the CD10-positive part (Figure $2 \mathrm{~b}(\mathrm{~A}-\mathrm{C})$ ), suggesting that ARPP may not be expressed in the proximal tubule, but rather it may in part of the distal tubule in the renal cortex. On the other hand, in the renal medulla, ARPP was found to be expressed selectively in part of the renal tubule composed of low cuboidal epithelial cells whose morphologic features apparently differ from those of the collecting duct (Figure 2a(D, E)). Double immunohistochemistry with anti-ARPP polyclonal $\mathrm{Ab}$ and anti-MUC1 Ab, which is known to be immunoreactive with the distal tubule and collecting duct, revealed that the ARPP-positive part of the renal 
a
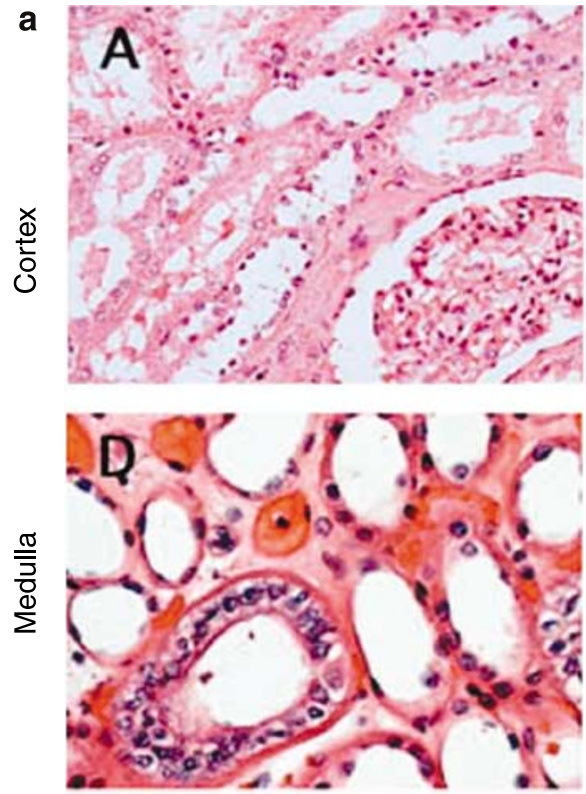

b
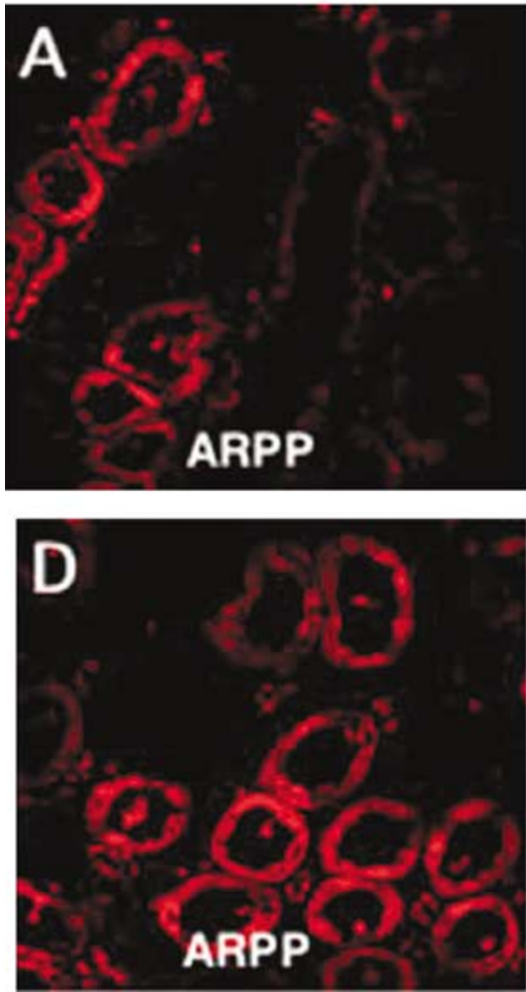
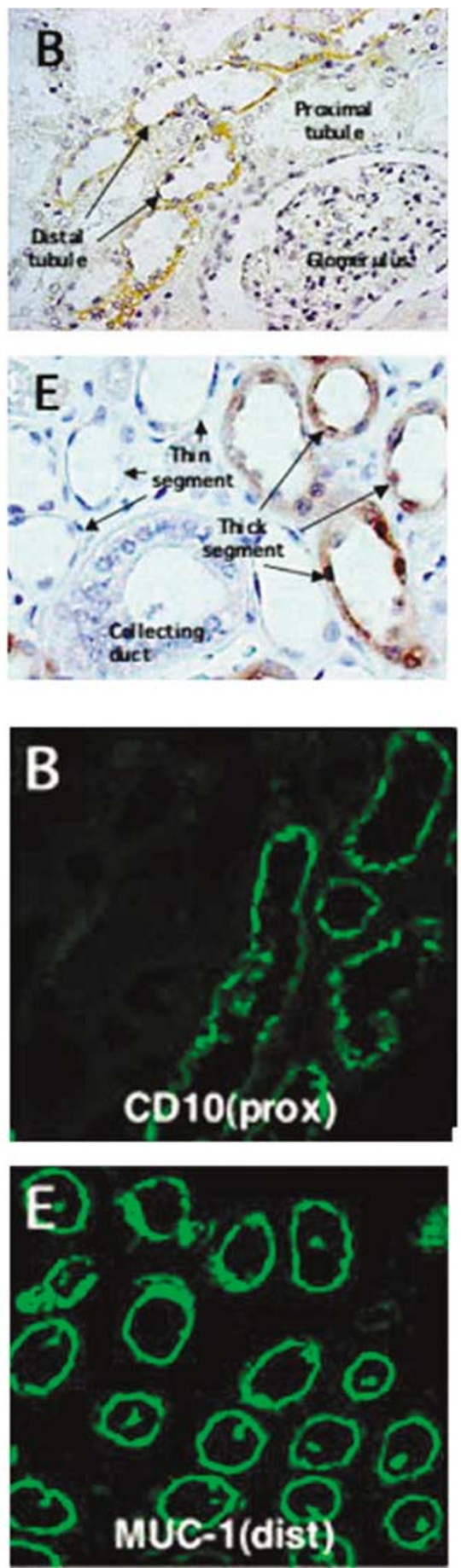
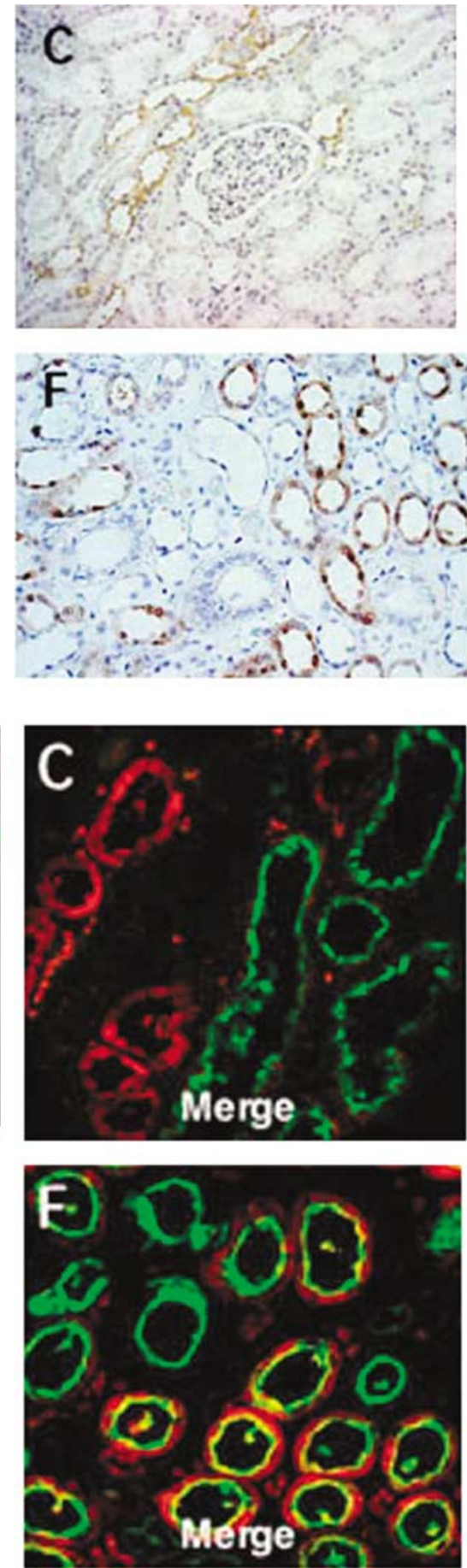

Figure 2 Expression of ARPP in normal kidney. (a) Immunohistochemical analysis of ARPP in normal kidney. Tissue sections prepared from paraffin-embedded normal kidney tissue were deparaffinized and immunostained with YAS-11 Ab (B, C, E, and F). Serial sections were stained with hematoxylin and eosin (A and D). Views at high magnification $(\times 40 ; \mathrm{A}, \mathrm{B}, \mathrm{D}$, and E) and views at low magnification $(\times 10$; $\mathrm{C}$ and $\mathrm{F})$ are shown. In the renal cortex, part of the distal renal tubule with low cuboidal epithelium was found to be immunostained with YAS-11 Ab (B), but the proximal renal tubule (arrows in C) and collecting duct (arrowheads in E, F) were not stained. (b) Double-labeled immunofluorescense of normal kidney. Tissues sections of normal kidney were incubated with a mixture of YAS-11 Ab and either CD10 Ab or MUC-1 Ab. After being washed with PBS, the sections were incubated with a mixture of Alexa Fluor 488-conjugated goat anti-rabbit antibody and Alexa Fluor 546-conjugated goat anti-mouse antibody in $1 \times$ PBS. Consequently, ARPP was detected as red signals, as shown in A, C, D, and E. CD10 was detected as green signals, as shown in B and C. MUC-1 was detected as green signals, as shown in E and F. Merged signals were detected as yellow, as shown in F.

tubule was always positive for MUC1 (Figure 2b(DF)). Conversely, we were also able to detect MUC-1positive but ARPP-negative parts of the renal tubule
(Figure $2 b(D-F))$. These findings suggest that ARPP may be expressed in part of the distal renal tubule in both the renal cortex and medulla. 


\section{ARPP is Rarely Expressed in RCCs but Selectively Expressed in Oncocytomas}

Next, we immunohistochemically analyzed 14 oncocytomas and 86 RCCs for expression of ARPP using paraffin-embedded tissue sections. As shown in Figure 3 and Table 1, ARPP was expressed in 12 of the 14 oncocytomas (Table 1, Figure 3B), two $(5.1 \%)$ of the 39 cases of the eosinophilic variant of clear cell RCC, and two (28.6\%) of seven cases of papillary RCC (two positive tumors were Type I). On the other hand, ARPP was not detected in any of the chromophobe RCCs or clear cell RCCs (Table 1, Figure $3 \mathrm{~F}, \mathrm{~J}$, and $\mathrm{N}$ ), suggesting that ARPP is selectively expressed in oncocytomas but rarely expressed in other types of renal tumor. Thus, it appears that ARPP could have potential use as a marker for distinguishing oncocytomas from chromophobe RCCs.

We then compared the expression of ARPP with that of other existing markers including EMA and CD10. Immunohistochemical analysis of 100 renal tumors including 14 oncocytomas and 86 conventional RCCs revealed that CD10, a marker for the proximal renal tubule, was expressed in $12(85.7 \%)$ of the 14 oncocytomas, one $(9.1 \%)$ of the 11 chromophobe RCCs, 14 (56.0\%) of the 25 clear cell RCCs, 27 $(69.2 \%)$ of the 39 cases of the eosinophilic variant of clear cell RCC, and three (42.9\%) of the seven papillary RCCs (Table 1). These findings suggest that CD10 tends to be frequently expressed in every subtype of renal tumor except for chromophobe
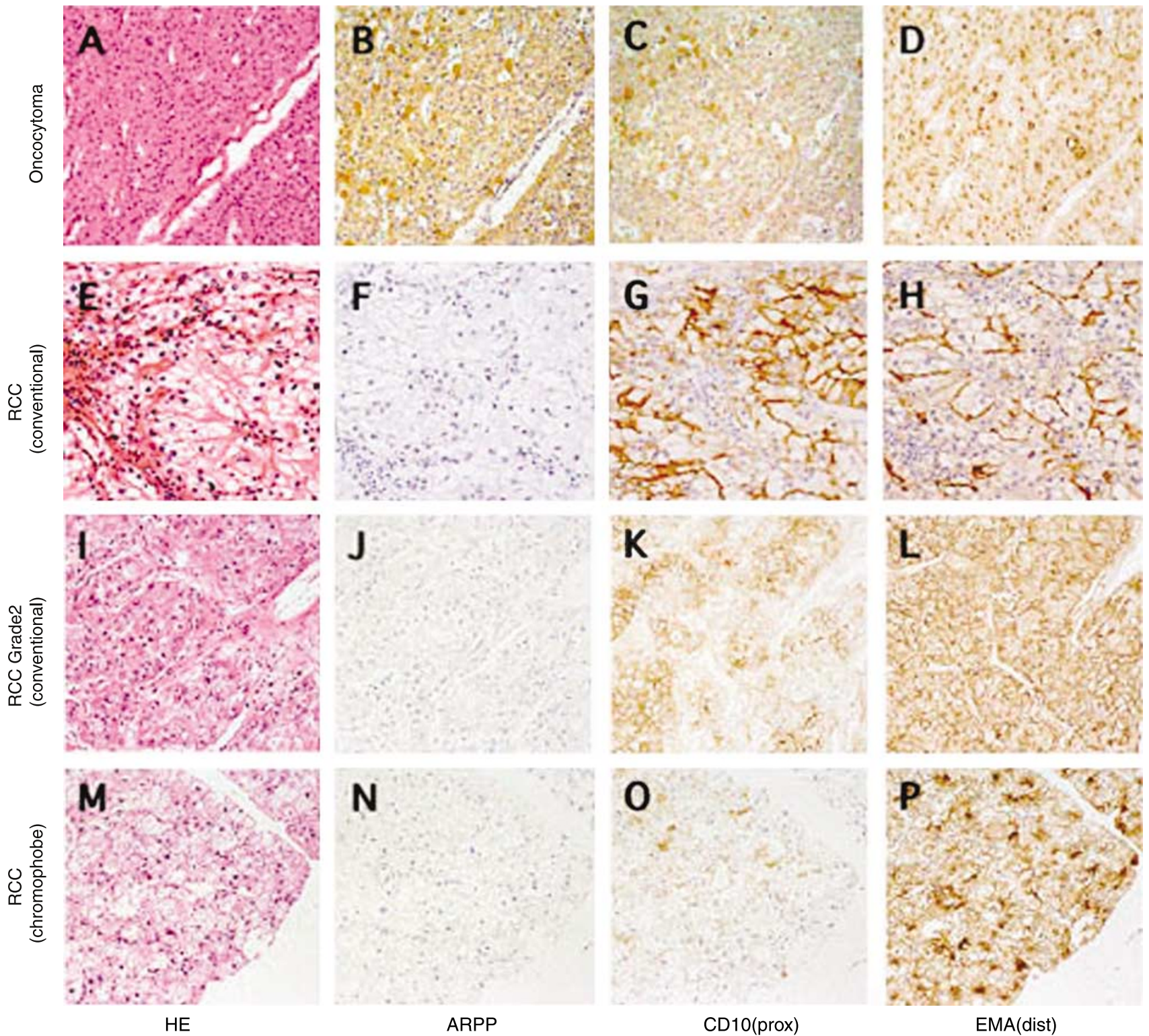

EMA(dist)

Figure 3 Specific expression of ARPP in renal oncocytoma. Serial tissue sections of renal oncocytoma (A-D), conventional RCC (Grade 1) (E-H), conventional RCC (Grade 2) (I-L), and chromophobe RCC (M-P) were immunostained with YAS-11 Ab (B, F, J, and N), anti-CD10 antibody (C, G, K, and $\mathbf{O}$ ), and anti-MUC-1 antibody (D, H, L, and $\mathbf{P}$ ), accompanied by routine HE staining (A, E, I, and $\mathbf{M}$ ). 
Table 1 Immunoreactivity for ARPP, EMA, and CD10 in renal tumors

\begin{tabular}{lccc}
\hline Renal tumors & ARPP & EMA & CD10 \\
\hline Oncocytoma & $85.7 \%(12 / 14)$ & $85.7 \%(12 / 14)$ & $50.0 \%(7 / 14)$ \\
& & & \\
Renal cell carcinoma (RCC) & $4.7 \%(4 / 86)$ & $86.0 \%(74 / 86)$ & $54.9 \%(45 / 82)$ \\
Chromophobe RCC & $0 \%(0 / 11)$ & $96.0 \%(11 / 11)$ & $9.1 \%(1 / 11)$ \\
Clear cell RCC & $0 \%(0 / 29)$ & $92.3 \%(36 / 39)$ & $56.0 \%(14 / 25)$ \\
Clear cell RCC (eosinophilic) & $5.1 \%(2 / 39)$ & $42.9 \%(3 / 7)$ & $69.2 \%(27 / 39)$ \\
Papillary RCC & $28.6 \%(2 / 7)$ & $42.9 \%(3 / 7)$ \\
\hline
\end{tabular}

RCC. A marker for the distal renal tubule, EMA, was also analyzed for its expression in renal tumors. EMA was found to be expressed in $12(85.7 \%)$ of the 14 oncocytomas, all $(100 \%)$ of the 11 chromophobe RCCs, $24(96.0 \%)$ of the 29 clear cell RCCs, 36 $(92.3 \%)$ of the 39 cases of the eosinophilic variant of clear cell RCC, and three (42.9\%) of the seven cases of papillary RCC. Although EMA was found to be highly expressed in oncocytomas, it was also expressed in other subtypes. These results suggest that ARPP is expressed more specifically in oncocytomas than other existing markers for this tumor type.

\section{Ultrastructural Localization of ARPP in Normal Kidney and Renal Oncocytoma}

Next, to determine the ultrastructural localization of ARPP in normal kidney and oncocytoma, we performed immunoelectron microscopy with the anti-ARPP polyclonal Ab. As shown in Figure 4a, positive immunoreactivity was found in low cuboidal tubular epithelial cells. These cells have abundant mitochondria and short microvilli at the luminal surface, but lack a brush border, suggesting that ARPP-positive cells were unlikely to be located in the proximal renal tubule, but rather in the distal tubule. Further observations revealed that ARPP was localized in mitochondria and nuclei, indicating that ARPP is expressed specifically in these organelles in the distal renal tubule of the normal kidney (Figure 4a).

Next, we analyzed the ultrastructural localization of ARPP in oncocytoma. As shown in Figure 4b, strong immunoreactivity was confined to the mitochondria, and weaker signals were also detectable in the nuclei of some tumor cells (Figure 4b). These findings suggest that the ultrastructural localization of ARPP in the distal renal tubule is quite similar to that in oncocytoma. Interestingly, positive immunoreactivity for ARPP within mitochondria was not distributed diffusely, but tended to be concentrated at the periphery, both in oncocytoma and the distal renal tubule (Figure $4 \mathrm{~b}$ ).

\section{Discussion}

Renal oncocytoma is an unusual epithelial neoplasm that is considered benign. ${ }^{9,11}$ It is characterized
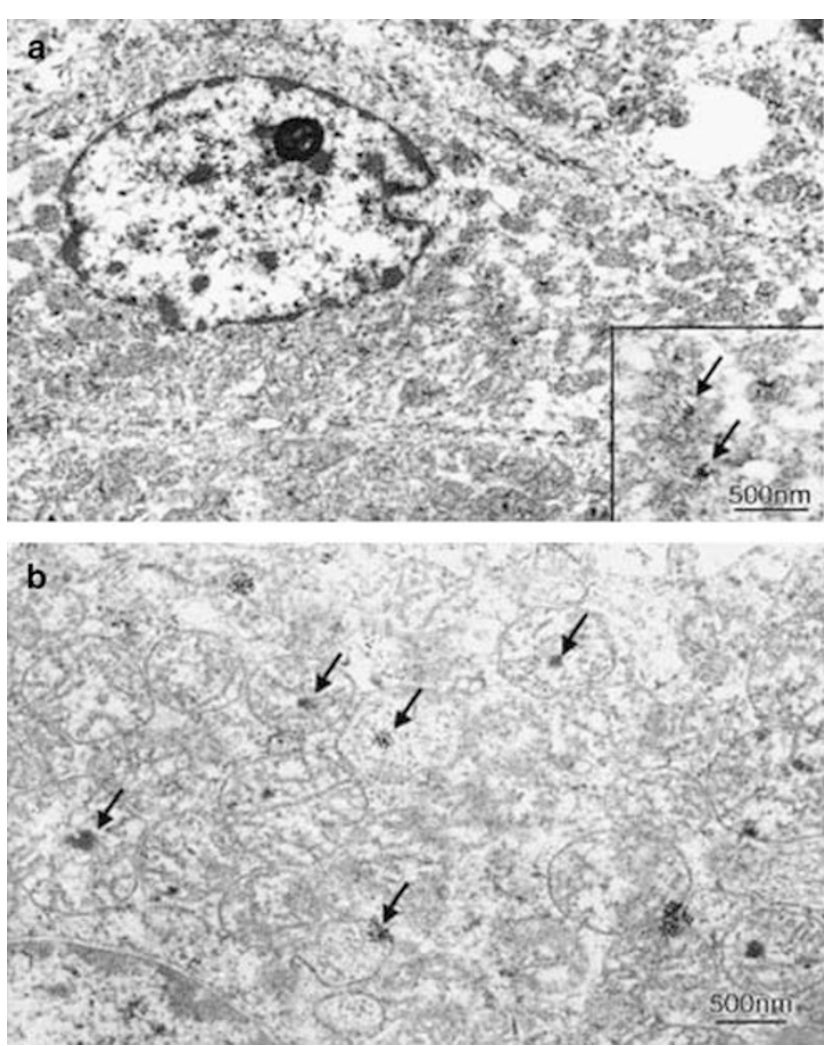

Figure 4 Immunoelectron microscopy for expression of ARPP in normal kidney and renal oncocytoma. Ultrastructural localization of ARPP in normal kidney tissue obtained at autopsy and in paraffin-embedded oncocytoma tissue obtained at surgery were analyzed by immunoelectron microscopy with anti-ARPP polyclonal antibody. In normal kidney (a), positive immunoreactivity was observed in mitochondria (arrows in a) and nuclei (arrowheads in b). In oncocytoma (b), strongly positive immunoreactivity was detected in mitochondria (b) (shown in the inset at high magnification).

histopathologically by diffuse proliferation of pinkred cells with abundant granular cytoplasm associated with a loose edematous stroma and prominent vasculature, and by absence of severe nuclear atypia, mitoses, macronucleoli, and clear cells. ${ }^{12-16}$ Although the renal oncocytoma from RCC is of prognostic significance, differential separation diagnosis between the two tumors is sometimes difficult, because both the eosinophilic variant of clear celltype RCC and chromophobe cell-type RCC are morphologically similar to renal oncocytoma. ${ }^{12,17}$ It 
has been reported that some tumor markers including vinculin, paxillin, SHP2, parvalbumin, and c-kit are highly expressed in chromophobe RCC and oncocytoma, but scarcely expressed in clear cell RCC and papillary RCC. ${ }^{18-24}$ Thus, it is evident that these existing markers can be used to separate chromophobe RCC and oncocytoma from conventional RCC. However, there are few reports of tumor markers that can be used to separate oncocytoma from chromophobe RCC. EMA, known to be a marker of the distal renal tubule and collecting duct, is reportedly expressed not only in $67-85.7 \%$ of oncocytomas but also $75-100 \%$ of chromophobe RCCs, ${ }^{25,26}$ suggesting that it is not useful for distinguishing between the two tumors. Furthermore, Carion et $a l^{27}$ have recently reported that, although caveolin is expressed in $91.7 \%$ of oncocytomas, it is also expressed in $26.9 \%$ of clear cell RCCs, $19.2 \%$ of papillary RCCs, and $21 \%$ of chromophobe RCCs. Although caveolin is expressed more frequently in oncocytoma than in chromophobe RCC, it is still difficult to separate the two neoplasms based on the expression pattern of caveolin alone. In this study, we found that ARPP was highly expressed in $12(85.7 \%)$ of 14 renal oncocytomas, whereas it was detectable in only four $(4.7 \%)$ of 86 conventional RCCs. It was noteworthy that none of 11 chromophobe RCCs expressed ARPP. Based on these findings, we propose that ARPP can be used as a specific marker for oncocytoma, and that it may be useful for differential diagnosis between oncocytoma and chromophobe RCC.

We reported previously that ARPP was localized at the sarcomeric I-bands as well as nuclei of skeletal muscle. ${ }^{5}$ On the other hand, in the kidney, we found in the present study that ARPP was localized in mitochondria and nuclei of distal renal tubule cells, suggesting that ARPP may have a specific functional role in these organelles in this part of the nephron. Interestingly, immunoelectron microscopy revealed that the immunoreactivity of ARPP within mitochondria was not distributed diffusely but in focal clusters. The biological significance of this characteristic localization pattern within mitochondria remains to be determined. Recently, we have developed ARPP gene-disrupted mice (unpublished data), and it is anticipated that analysis of these mice will help to clarify the biological and physiological role of ARPP in the kidney.

It has been believed that the cell origin of conventional renal cell carcinoma may be the renal proximal tubule, ${ }^{28,29}$ whereas it has been proposed that oncocytoma and chromophobe RCC are derived from the epithelium of the distal nephron, ${ }^{30,31}$ especially the intercalated cells of the collecting tubule. $^{32}$ Electron microscopic analysis of oncocytoma revealed the presence of round to oval mitochondria, sparse and blunt microvilli, extensive interdigitation and invagination of the basal plasmalemma, and protrusions of the basement membrane into concavities in the plasmalemma, thus resembling the morphologic features of distal nephron. $^{30}$ Furthermore, it has been reported that epithelial membrane antigen (EMA), carbonic anhydrase $\mathrm{C}$ (CAC) and Band 3, whose expression is restricted to the distal renal tubule, are exclusively expressed in oncocytoma. ${ }^{21,33,32,34-36}$ On the other hand, CD10, a marker of the proximal renal tubule, is not expressed in oncocytoma. ${ }^{33,37}$ Those previous reports support the notion that oncocytoma may be derived from the distal nephron. Our present immunohistochemical and immunoelectron microscopic studies revealed that ARPP expression in the kidney was confined to the mitochondria and nuclei of cells in the distal renal tubule, similar to the ARPP expression observed in oncocytoma. Thus, our present findings support the hypothesis that oncocytoma may arise from the part of the distal nephron that expresses ARPP.

\section{References}

1 Moriyama M, Tsukamoto Y, Fujiwara $\mathrm{M}$, et al. Identification of a novel human ankyrin-repeated protein homologous to CARP [abstract]. Biochem Biophys Res Comm 2001;285:715-723.

2 Kemp TJ, Sadusky TJ, Saltisi F, et al. Identification of Ankrd2, a novel skeletal muscle gene coding for a stretch-responsive ankyrin-repeat protein [abstract]. Genomics 2000;66:229-241.

3 Ishiguro N, Baba $\mathrm{T}$, Ishida $\mathrm{T}$, et al. Carp a cardiac ankyrin-repeated protein and its new homologue, Arpp are differentially expressed inheart skeletal muscle and rhabdomyosarcomas [abstract]. Am J Pathol 2002;160:1767-1778.

4 Ishiguro N, Motoi T, Osaki M, et al. Immunohistochemical analysis of a muscle ankyrin-repeat protein, Arpp, in paraffin-embedded tumors: evaluation of Arpp as a tumor marker for rhabdomyosarcomas [abstract]. Hum Pathol 2005;36:620-625.

5 Tsukamoto Y, Senda T, Nakano T, et al. Arpp, a new homolog of Carp is preferentially expressed in type 1 skeletal muscle fibers and is markedly induced by denervation [abstract]. Lab Invest 2002;82: 645-655.

6 Galfre G, Milstein C. Preparation of monoclonal antibodies: strategies and procedures [abstract]. Methods Enzymol 1981;73(Part B):3-46.

7 Ishida T, Tojo T, Aoki T, et al. TRAF5, a novel tumor necrosis factor receptor-associated factor family protein, mediates CD40 signaling [abstract]. Proc Natl Acad Sci USA 1996;93:9437-9442.

8 Engvall E. Enzyme immunoassay ELISA and EMIT [abstract]. Methods Enzymol 1980;70:419-439.

9 Reuter VE, Davis CJ, Moch H. Oncocytoma. In: Eble JN, Sauter G, Epstein JI (eds). World Health Organization Classification of Tumours. Pathology and Genetics of Tumours of the Urinary System and Male Genital Organs. IARC Press: Lyon, France, 2004, pp 42-43.

10 Yano S, Kashima K, Daa T, et al. An antigen retrieval method using an alkaline solution allows immunoelectron microscopic identification of secretory granules in conventional epoxy-embedded tissue sections [abstract]. J Histochem Cytochem 2003;51:199-204. 
11 Novick AC, Campbell SC. Renal tumors. In: Walsh PC (ed). Campbell's Urology: Renal Tumors, 8th edn. Saunders: New York, NY, 2003, pp 2672-2731.

12 Barnes CA, Beckman EN. Renal oncocytoma and its congeners [abstract]. Am J Clin Pathol 1983;79:312-318.

13 Chang A, Harawi SJ. Oncocytes, oncocytosis, and oncocytic tumors [abstract]. Pathol Annu 1992;27: 263-304.

14 Klein MJ, Valensi QJ. Proximal tubular adenomas of kidney with so-called oncocytic features. A clinicopathologic study of 13 cases of a rarely reported neoplasm [abstract]. Cancer 1976;38:906-914.

15 Merino MJ, Livolsi VA. Oncocytomas of the kidney [abstract]. Cancer 1982;50:1852-1856.

16 Weiss LM, Gelb AB, Medeiros LJ. Adult renal epithelial neoplasms [abstract]. Am J Clin Pathol 1995;103: 624-635.

17 Dabbs DJ. The surgical pathologist's approach to fine needle aspiration [abstract]. Clin Lab Med 1998;18: 357-372.

18 Kuroda N, Hayashi Y, Matozaki T, et al. Differential expression of SHP2, a protein-tyrosine phosphatase with SRC homology-2 domains, in various types of renal tumor [abstract]. Virchows Arch 1998;433: 331-339.

19 Kuroda N, Naruse K, Miyazaki E, et al. Vinculin: its possible use as a marker of normal collecting ducts and renal neoplasms with collecting duct system phenotype [abstract]. Mod Pathol 2000;13:1109-1114.

20 Kuroda N, Guo L, Toi M, et al. Paxillin: application of immunohistochemistry to the diagnosis of chromophobe renal cell carcinoma and oncocytoma [abstract]. Appl Immunohistochem Mol Morphol 2001;9:315-318.

21 Kuroda N, Toi M, Hiroi M, et al. Review of chromophobe renal cell carcinoma with focus on clinical and pathobiological aspects [abstract]. Histol Histopathol 2003;18:165-171.

22 Martignoni G, Pea M, Chilosi M, et al. Parvalbumin is constantly expressed in chromophobe renal cell carcinoma [abstract]. Mod Pathol 2001;14:760-767.

23 Petit A, Castillo M, Santos M, et al. KIT expression in chromophobe renal cell carcinoma: comparative immunohistochemical analysis of KIT expression in different renal cell neoplasms [abstract]. Am J Surg Pathol 2004;28:676-678.

24 Pan CC, Chen PC, Chiang H. Overexpression of KIT (CD117) in chromophobe renal cell carcinoma and renal oncocytoma [abstract]. Am J Clin Pathol 2004; 121:878-883.
25 Abrahams NA, MacLennan GT, Khoury JD, et al. Chromophobe renal cell carcinoma: a comparative study of histological, immunohistochemical and ultrastructural features using high throughput tissue microarray [abstract]. Histopathology 2004;45:593-602.

26 Khoury JD, Abrahams NA, Levin HS, et al. The utility of epithelial membrane antigen and vimentin in the diagnosis of chromophobe renal cell carcinoma [abstract]. Ann Diagn Pathol 2002;6:154-158.

27 Carrion R, Morgan BE, Tannenbaum M, et al. Caveolin expression in adult renal tumors [abstract]. Urol Oncol 2003;21:191-196.

28 Oberling C, Riviere M, Haguenau F. Ultrastructure of the clear cells in renal carcinomas and its importance for the demonstration of their renal origin [abstract]. Nature 1960;186:402-403.

29 Wallace AC, Nairn RC. Renal tubular antigens in kidney tumors [abstract]. Cancer 1972;29:977-981.

30 Eble JN, Hull MT. Morphologic features of renal oncocytoma. A light and electron microscopic study [abstract]. Human Pathol 1984;15:1054-1061.

31 Otani M, Shimizu T, Serizawa H, et al. Low-grade renal cell carcinoma arising from the lower nephron: a case report with immunohistochemical, histochemical and ultrastructural studies [abstract]. Pathol Int 2001;51: 954-960.

32 Storkel S, Pannen B, Thoenes W, et al. Intercalated cells as a probable source for the development of renal oncocytoma [abstract]. Virchow Archiv B 1988 56:185-189.

33 Nagashima Y. Chromophobe renal cell carcinoma: clinical, pathological and molecular biological aspects [abstract]. Pathol Int 2000;50:872-878.

34 Drenckhahn D, Schluter K, Allen DP, et al. Colocalization of Band 3 with ankyrin and spectrin at the basal membrane of intercalated cells in the rat kidney [abstract]. Science 1985;230:1287-1289.

35 Ortmann M, Vierbuchen M, Fischer R. Renal oncocytoma: II. Lectin and immunohistochemical features indicating an origin from the collecting duct [abstract]. Virchow Arch B 1988;56:175-184.

36 Storkel S, Steart PV, Drenkhahn D, et al. The human chromophobe cell renal carcinoma: its probable relation to intercalated cells of the collecting duct [abstract]. Virchow Arch B 1989;56:237-245.

37 Avery AK, Beckstead J, Renshaw AA, et al. Use of antibodies to RCC and CD10 in the differential diagnosis of renal neoplasms [abstract]. Am J Surg Pathol 2000;24:203-210. 